livraisons

d'Histoire

de l'Architecture

\section{Livraisons de l'histoire de l'architecture}

$39 \mid 2020$

Maquettes d'architecture

\title{
Concours de beauté : maquettes et photographies de maquettes aux Etats-Unis
}

Beauty contests: models and model photography in the United States

Schönheitswettbewerbe. Architekturmodelle und deren Photographien in den

Vereinigten Staaten

\section{Teresa Fankhänel}

\section{(2) OpenEdition}

\section{Journals}

Édition électronique

URL : http://journals.openedition.org//ha/1551

DOI : 10.4000//ha.1551

ISSN : 1960-5994

Éditeur

Association Livraisons d'histoire de l'architecture - LHA

Édition imprimée

Date de publication : 15 juin 2020

Pagination : 27-42

ISSN : 1627-4970

\section{Référence électronique}

Teresa Fankhänel, «Concours de beauté : maquettes et photographies de maquettes aux Etats-Unis », Livraisons de l'histoire de l'architecture [En ligne], 39 | 2020, mis en ligne le 24 janvier 2021, consulté le 26 janvier 2021. URL : http://journals.openedition.org//ha/1551 ; DOl : https://doi.org/10.4000//ha. 1551 
Par Teresa FANKHÄNEL

\section{CONCOURS DE BEAUTÉ : MAQUETTES ET PHOTOGRAPHIES DE MAQUETTES AUX ÉTATS-UNIS ${ }^{1}$}

\section{La laideur du Rockefeller Center}

Le 6 mars 1931, une photo du New York Times (ill. 1)

«a fait réagir le public comme aucune autre entreprise architecturale ne l'avait jamais fait et a donné le mois dernier aux New-Yorkais, un sujet de conversation controversé qui dura bien au-delà des neuf jours habituels de publicité des journaux. ${ }^{2}$

L'image contestée était une photographie en noir et blanc d'une maquette en plâtre du projet de Rockefeller Center qui était sur le point de transformer le centreville de Manhattan d'une aire résidentielle et commerciale en un nouveau centre-ville. La maquette représentait les nouvelles structures comme de simples masses géométriques avec des fenêtres sculptées sur l'extérieur, sans aucune autre forme d'embellissement. Vus depuis un angle élevé en surplomb, les bords du socle de la maquette et donc du site du chantier étaient clairement visibles sur la photographie, affichant un objet de petite échelle. Même si le Times avait assuré à ses lecteurs que «les intérieurs sont somptueux " et que le bâtiment ovale faisant face à la Cinquième Avenue ressemblait à un "poudrier orné de pierres précieuses sur une coiffeuse " ${ }^{3}$, les critiques du projet n'étaient pas moins fleuries dans leurs dénonciations, qualifiant le même bâtiment de "boîte à chapeau ", ou pire encore, de «burette d'huile ", un coup bas plutôt personnel à l'attention de John D. Rockefeller étant donné son implication avec Standard Oil. Avec le recul, le tollé général suscité par la laideur présumée du Rockefeller Center n'était peut-être que partiellement dû à son aspect lisse, moderne. Au-delà de la controverse, un bouleversement plus fondamental dans l'utilisation des dispositifs médiatiques architecturaux pour les présentations au public, était en cours. Défiant les conventions des propositions architecturales, la maquette du Rockefeller Center et ses photographies représentaient une nouveauté isolée et, par conséquent, mal comprise, exacerbée par le fait que le New York Times

1. Ce texte a été traduit de l'anglais par Bérénice Gaussuin et Stéphanie Quantin-Biancalani.

2. "Plan and Two Views of Model of Design for Radio City, Fifth Avenue, New York ", Pencil Points, mai 1931, 387.

3. «Radio City to Create a New Architecture ", The New York Times, 6 mars 1931. 


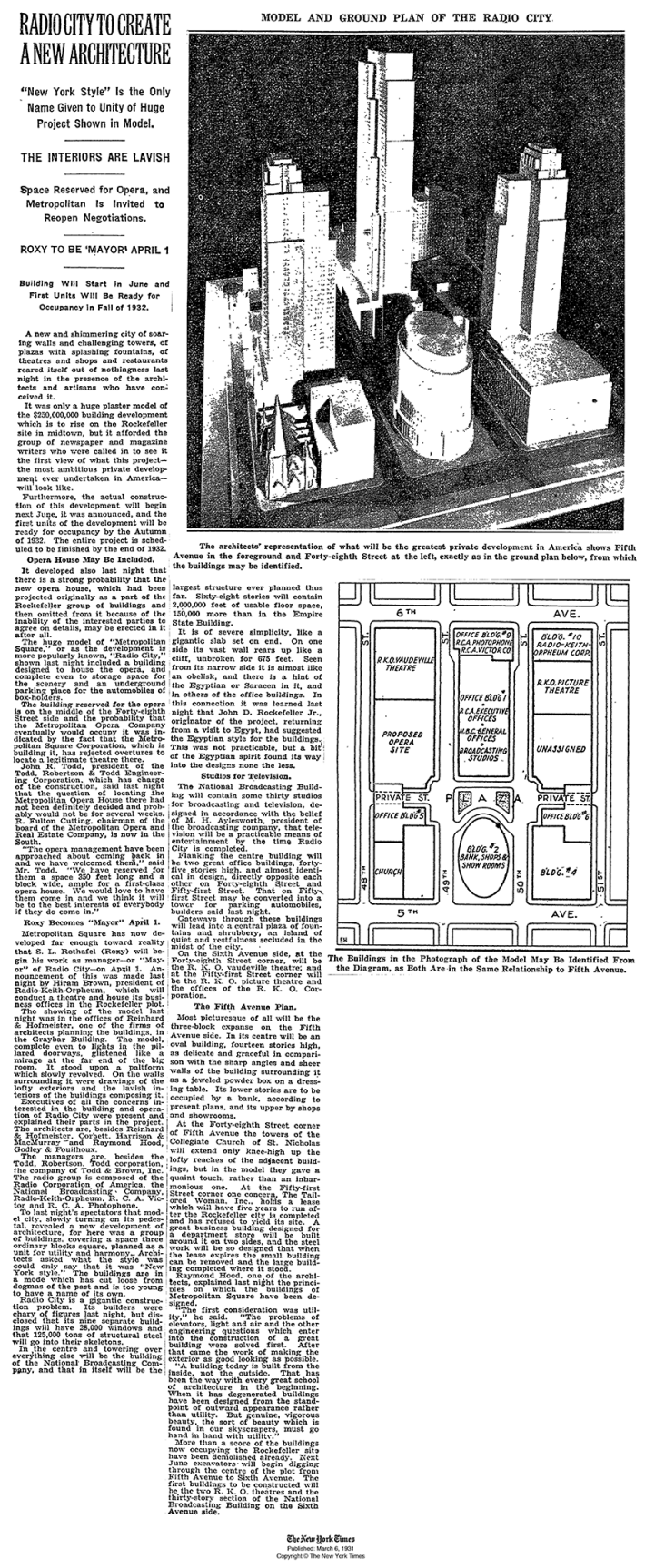

Ill. 1 : Maquette du Rockefeller Center publiée dans le New York Times, 6 mars 1931. (C) New York Times. 
ne reproduisit pas les dessins de grand format présentés aux journalistes lors de la conférence de presse ${ }^{4}$.

Bien qu'ils aient certainement existé au début du $\mathrm{XX}^{\mathrm{e}}$ siècle, les maquettes tout comme les photographies de maquettes n'étaient que rarement employées pour communiquer sans intermédiaire avec un public plus large. Un lent changement avait été enclenché au cours de la décennie précédant les plans du Rockefeller Center, lorsque certains architectes commencèrent à préférer aux rendus les maquettes, suscitant un débat enflammé, résumé au mieux par les mots-clés «feindre " contre " honnêteté ". Selon leurs défenseurs, les maquettes étaient plus adaptées et " honnêtes " car elles étaient plus à même de transmettre une idée tridimensionnelle d'un bâtiment que des dessins qui étaient souvent "faux ", mettant de côté les vues défavorables ou embellissant un projet ${ }^{5}$. Les rendus avaient considérablement évolué au cours des années 1920, lorsque de nouvelles techniques de clair-obscur avait été introduites par des professionnels du rendu, tels que Hugh Ferriss, le perspectiviste le plus connu d'Amérique. Tournant le dos aux vues multiples rassemblées sur les grandes feuilles des panneaux de présentation dans la tradition des Beaux-Arts, qui comprenaient souvent des plans d'étage et des perspectives colorées, particulièrement raffinées, les nouveaux rendus étaient une interprétation plutôt qu'une reproduction exacte d'un bâtiment. Les dessins au fusain très séduisants de Ferriss présentaient une vision dramatique des masses d'un bâtiment plutôt qu'un aperçu détaillé, et il s'intéressait plus à l'« essence " d'un bâtiment qu'à la précision. Ses images étaient souvent dessinées avec de puissants jeux d'ombre et de lumière, à l'exclusion de tous les détails - un exemple typique pour les promoteurs de la maquette qui dénonçaient ces dessins comme des "faux ». Pourtant, quelque peu en contradiction avec leur critique des dessins, les mêmes rendus en clair-obscur ont rapidement été privilégiés pour d'importantes commandes et des présentations publiques par un certain nombre d'architectes progressistes, parmi lesquels les défenseurs des maquettes et architectes du Rockefeller Center, Harvey Wiley Corbett et Raymond Hood.

Jusque-là, la fabrication de maquettes architecturales reposait sur une myriade de matériaux - carton, bois, argile et plâtre - pour ne nommer que les plus couramment utilisés. Chacun d'eux présentait ses propres avantages dans la production d'objets à petite échelle. Le carton était bon marché, léger, facile à utiliser avec des outils à main et pouvait être peint à l'aquarelle. Le bois était plus solide, mais exigeait que l'architecte réserve une pièce séparée dans l'agence à cause de la poussière et des copeaux de bois fins. Le plâtre et l'argile se prêtaient au travail sculptural, mais nécessitaient souvent l'intervention d'un plâtrier professionnel. Des architectes comme Corbett et Hood ont été parmi les premiers à New York à avoir largement recours aux maquettes pour leurs pratiques de conception et de présentation, chacun spécialisé dans un matériau : Corbett préféra le carton et Hood se décida pour l'argile et le plâtre. L'introduction de la Zoning Law de New York de 1916, qui

4. Ibid.

5. Simon Breines, "Paper Architecture", The American Architect, avril 1931, p. 25. 
obligeait les architectes à commencer la conception des structures hautes en déterminant d'abord la plus grande masse possible permise par le nouveau règlement, contribua à renforcer l'intérêt pour les maquettes. De là, les maquettes sont devenues un outil agréable d'expérimentation sur les enveloppes des bâtiments qui pouvaient être adaptées au fur et à mesure de la conception pour intégrer le pourtour de la façade. Le traitement extérieur de ces maquettes dépendait largement du matériau de fabrication ; mais, le plus souvent, les façades étaient dessinées ou peintes sur la surface des maquettes à petite échelle. Par rapport aux rendus qui se limitaient à la présentation d'un angle à la fois, l'un de leurs plus grands avantages était de fournir une variété de perspectives, d'éclairage et de cadrage, en particulier dans leur traduction en photographies de maquettes.

Les photographies de maquettes ont une histoire tout aussi longue, leur utilisation datant de l'invention de la photographie au XIX siècle $^{6}$. Dans les années 1920, leur usage oscillait entre les besoins en conception, les aides pour les rendus en perspective et les présentations semi-privées aux clients. Elles étaient rarement reproduites dans des publications d'intérêt général, des revues d'architecture ou des journaux. De manière générale, le principal médium pour présenter un projet non construit dans une publication restait le rendu. Tout comme les objets avec lesquels elles ont été réalisées, les photographies ont traversé une période intense d'expérimentation dans les années 1920 et 1930, sous l'influence forte d'autres dispositifs médiatiques prédominants pour les présentations architecturales : les rendus et la photographie d'architecture. Hormis les images documentaires qui consignaient des maquettes en tant qu'objets, la photographie en clair-obscur inspirée des rendus novateurs de Hugh Ferriss était une des principales représentations de maquettes qui transformaient les objets en bâtiments par le recours à de puissants jeux d'ombre et de lumière, mais en éliminant tous les détails ${ }^{7}$. Cette technique, qui convenait à la définition des masses d'un bâtiment après l'adoption de la Zoning Law, fut largement utilisée pour la représentation photographique des maquettes d'architecture. Au moment où la controverse du Rockefeller Center eut lieu en 1931, la première et principale fonction de la plupart des photographies de maquettes, en plus de documenter le projet, était de mettre en évidence les volumes d'un bâtiment et aussi de masquer la matérialité de la maquette. Les photographies de la maquette du Rockefeller Center combinèrent à la fois la méthode répandue du clair-obscur et une approche documentaire, traitant la maquette comme un objet et non comme un bâtiment, et manquaient du raffinement et de l'illusion des rendus en dévoilant son échelle relativement petite. Pour des architectes comme Corbett et Hood, elles étaient une suite logique de leur travail qui associait maquettes de conception et rendus de présentation en clair-obscur, puisqu'elles transcrivaient les effets de la maquette et du rendu en photographies de maquette. Le public avait des attentes pourtant différentes : leur regard formé aux rendus de style Beaux-Arts et étranger aux maquettes aux masses trop simplistes, l'amena à rejeter le projet.

6. Karen Moon, Modeling Messages, New York, Monacelli Press, 2005, p. 67.

7. Andrew Higgott et Timothy Wray, Camera Constructs, Farnham, Ashgate, 2012, p. 5. 
Pour lui, l'écart entre ce qu'une maquette pouvait représenter et ce qu'on pouvait attendre d'une photographie ou d'un rendu était trop divergent.

\section{La maison de verre de Collier's : "Real as Life and Just as Beautiful "}

Le 6 mai 1939, presque exactement huit ans après le fiasco du Rockefeller Center, le magazine lifestyle Collier's (ill. 2) publiait une série de photographies de maquettes d'une maison de verre conçue par Landefeld et Hatch, alors en construction sur le site de l'Exposition universelle de New York de 1939 à Flushing dans le Queens. Cette fois, les photographies étaient accompagnées d'une légende qui disait :

«La maison de verre qui fait parler d'elle à l'Exposition universelle sera terminée vers la mi-mai. Nous ne pouvions pas vous laisser attendre. Voici donc une maquette de Collier's, aussi réelle et belle que dans la vraie vie. ${ }^{8}$

Loin de vouloir réveiller des souvenirs de la fureur publique antérieure, les photographies de la maquette de Collier's nécessitaient désormais une déclaration pour s'assurer que les lecteurs ne les confondent pas avec le bâtiment lui-même. Beaucoup de choses avaient changé. En 1936, deux sociétés, Rohm \& Haas et DuPont,

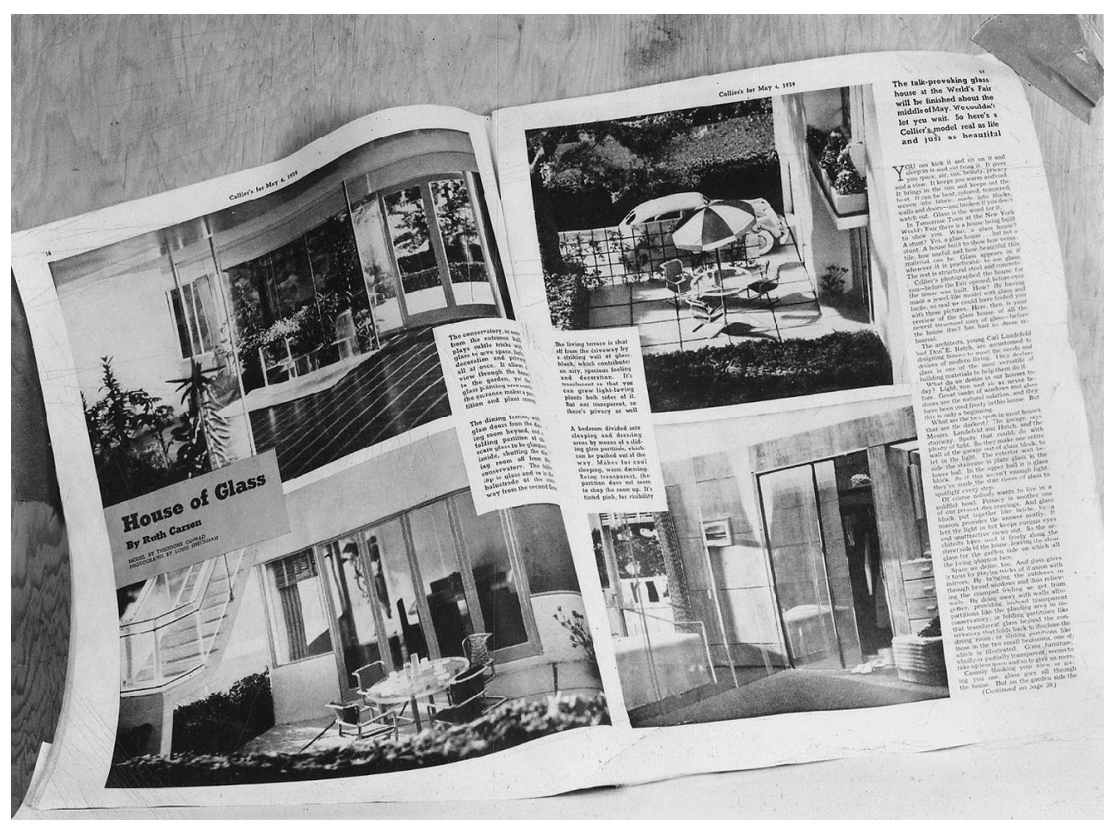

Ill. 2 : Double page originale du magazine Collier's, numéro du 6 mai 1939 présentant la maison de verre. (C) Avery Architectural and Fine Arts Library, Archives Theodore Conrad.

8. Ruth Carson, "House of Glass ", Collier's, 6 mai 1939, p. 17. 
avaient chacune de leur côté introduit un plastique translucide sur le marché américain sous les marques Plexiglas et Lucite 9 . Même si le matériau n'attira pas l'attention des designers industriels, il toucha presque immédiatement une corde sensible chez les maquettistes d'architecture devenus une profession indépendante au début des années 1930. Habitués à s'approprier une variété de matériaux de fabrication, ils étaient bien conscients d'une fracture croissante entre les développements des matériaux de construction et leur capacité limitée à les représenter avec les matériaux disponibles pour la fabrication de maquettes. Cet effet était également renforcé par l'introduction de grandes surfaces vitrées et les cadres en acier laissés apparents dans l'architecture moderne ; aucun des matériaux opaques précédents n'étaient en mesure de transmettre de manière adéquate le nouvel intérêt architectural pour la transparence et la connexion entre l'intérieur et l'extérieur d'un bâtiment.

Avec l'arrivée du plexiglas, c'est surtout la transparence du matériau qui a initialement séduit les maquettistes ${ }^{10}$. Mais ils ont rapidement découvert toutes les autres caractéristiques qui étaient aussi favorables à la fabrication de maquettes : le plexiglas pouvait être à la fois découpé et moulé. Il était léger, incassable et pouvait être peint ou collé sans apprêt. En quelques années seulement, les maquettistes d'architecture ont adapté le matériau pour représenter des surfaces transparentes et pour ses avantages structurels, qui permettaient l'assemblage des maquettes presque exclusivement à partir de ce matériau. Cela a conduit à des représentations des matériaux de construction plus précises que ce qui était possible auparavant, soit en utilisant le matériau non peint pour figurer le verre, soit en appliquant, par pulvérisation, des peintures élaborées pour les extérieurs. De manière concomitante, la construction de maquettes est passée de boîtes en carton pliées ou de volumes sculptés à des miniatures assemblées qui reproduisaient chacune des parties des bâtiments, jusqu'aux vitrages et menuiseries. Comme le plexiglas était beaucoup plus solide, les maquettes sont devenues plus grandes et ont pu représenter des intérieurs entièrement meublés visibles à travers les fenêtres transparentes. Sur la base de ces nouveaux matériaux, les photographies de maquettes ont commencé à gagner lentement en popularité. Sous les auspices d'un enthousiasme croissant pour le réalisme photographique, elles ont commencé à supplanter les rendus dans les publications architecturales et en particulier dans les magazines dédiés à l'art de vivre. Pour ces images, le réalisme signifiait la simulation de la réalité construite, une volonté conjointe des architectes et des maquettistes « de présenter leurs maquettes comme si elles étaient des bâtiments " ${ }^{11}$. Des tentatives similaires avaient été menées par Corbett, l'architecte du Rockefeller Center, avec ses maquettes en carton à par-

9. " 1936 Lucite ", 2.dupont.com, [dernière consultation 31 mars 2015] http://www2.dupont.com/ Phoenix_Heritage/en_US/1936_detail.html.

10. Hal Borland, "Worlds in Miniature for the Fair ", The New York Times, 12 mars 1939, p. 15.

11. Davide Deriu, "Transforming Ideas into Pictures. Model Photography and Modern Architecture ", Camera Constructs: Architecture and the Modern City, Andrew Higgott et Timothy Wray (dir.), Farnham, Ashgate, 2012, p. 161-175 et Mark Morris, "Worlds Collide: Reality to Model to Reality ", Camera Constructs : Architecture and the Modern City, Andrew Higgott et Timothy Wray (dir.), Farnham, Ashgate, 2012, p. 182. 
tir du début des années 1920. Dans un article publié dans l'Architectural Forum en 1922, il avait souligné le but ultime de la photographie de maquette :

«Ce que nous voulons vraiment savoir et montrer au client, c'est à quoi ressemblera le bâtiment vu comme la plupart des gens le verront, de la rue et à une faible distance. " ${ }^{12}$

Ce n'est qu'avec l'introduction des nouveaux matériaux qu'il a été possible de tenir cette promesse. Pour atteindre l'objectif de réalisme dans les photographies de maquettes, une précision maximale était une condition préalable à la fabrication de la maquette, car la moindre erreur détruirait l'illusion en rendant visibles les matériaux de la maquette et sa fabrication. En même temps, la maquette devait être à la fois détaillée et suffisamment grande pour supporter les vues en gros plan. Ainsi, les photographies devaient être le fruit d'un équilibre entre le désir de cacher la vraie nature de la maquette en tant qu'objet à petite échelle et le besoin accru de montrer des matériaux de construction tels que le verre transparent et le métal réfléchissant. La vraie taille et la matérialité de la maquette étaient dissimulées par des points de vue bas, "humains ", introduisant une simulation d'échelle à l'aide d'accessoires tels que des voitures ou des arbres. L'éclairage, les ombres et les arrièreplans renforçaient la simulation en imitant la lumière naturelle. À plus grande échelle, les maquettes peuvent être démontées, ce qui permettait à l'objectif photographique d'accéder à l'intérieur.

En 1939, les photographies de Louis Checkman de la maquette de la Maison du verre réalisée par Theodore Conrad (ill. 3) ont été parmi les premières à atteindre

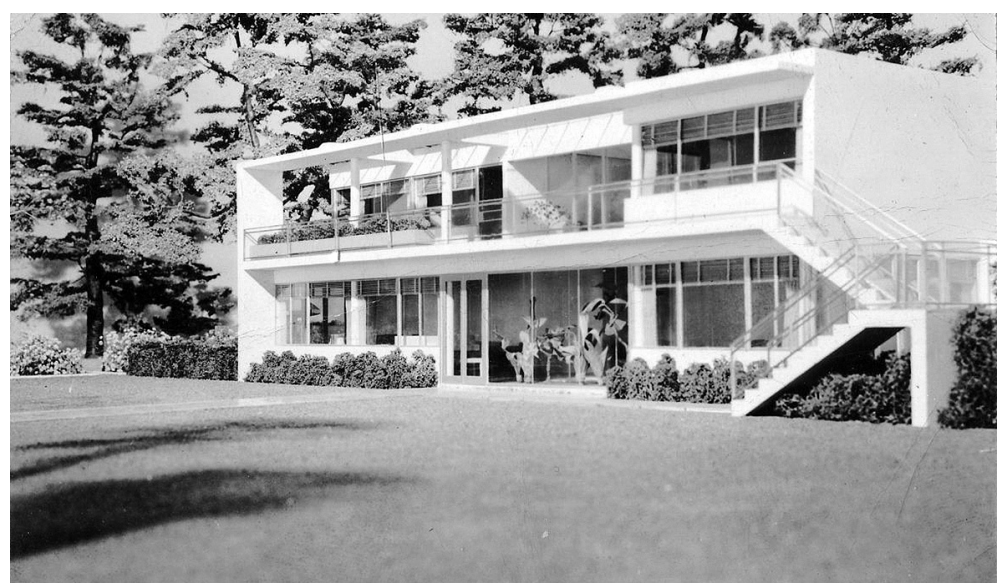

Ill. 3 : Photographie de l'extérieur de la maquette de la maison de verre. (C) Avery Architectural and Fine Arts Library, Archives Theodore Conrad.

12. Harvey Wiley Corbett, "Architectural Models of Cardboard ", Pencil Points, juillet 1922, p. 15. 
un niveau de réalisme jusqu'alors inégalé. Sans doute l'exemple le plus réussi de photographies de magazines d'avant-guerre, ces images montraient, comme jamais auparavant, des prises de vue intérieures et extérieures, à hauteur d'œil, d'une maquette meublée et détaillée. Les intérieurs étaient particulièrement exceptionnels de réalisme, jusqu'à l'éclat du verre, les rayons du soleil au sol et les reflets des fenêtres. Nouveauté récemment introduite, la toile de fond était légère et aérée, tandis que le puissant clair-obscur des années 1920 était abandonné au profit d'une lumière plus uniforme pour faire ressortir les matériaux, la transparence et les détails de la maquette. Le cadrage serré de l'image coupait les bords de la maquette qui étaient assombris par des plantes artificielles placées à l'extérieur du socle de la maquette. À la différence du Rockefeller Center, cette maquette offrait un aperçu précis du bâtiment, qui recréait sur les photographies la principale prouesse de la maison de verre en taille réelle, c'est-à-dire sa translucidité. Construite et publiée pour un grand public qui, jusqu'à récemment, avait rejeté la présentation d'un bâtiment par photographies de maquettes, elle a préparé le terrain pour un boom de photographies de maquettes similaires qui sont revenues à des contextes plus strictement architecturaux après la guerre, modifiant plus en profondeur la conception architecturale.

\section{L'ère des magazines}

En 1956, le maquettiste Theodore Conrad relata le raisonnement qui sous-tendait la recrudescence des photographies de maquettes destinées à donner un aperçu du bâtiment, en évoquant sa maquette de la maison réalisée par Gregory Ain pour le jardin du Museum of Modern Art de New York (ill. 4) :

"Il faut environ trois mois pour imprimer des pages en couleur dans un magazine. Si une maison est terminée en hiver, elle ne peut pas être photographiée avant le printemps. [...] Dans le cas de cette maison, elle a été construite dans le jardin du Museum of Modern Art et terminée au printemps. Le jour de son ouverture au public, elle avait l'air d'une couverture pour un magazine de premier plan. La maquette a dû être photographiée en janvier pour respecter cette date de publication. " ${ }^{13}$

Au milieu des années 1930, les photographies de maquettes devenaient le moyen de donner un aperçu au public des structures projetées, notamment pour des projets à grande échelle tels que l'Exposition universelle de New York, qui diffusa un nombre sans précédent de photographies de maquettes dans des communiqués de presse. Pourtant, jusqu'à la fin des années 1940, les photographies de maquettes réalistes restèrent largement dans le domaine des magazines lifestyle et féminins, dépeignant souvent de fictives maisons unifamiliales modernes, précurseures des

13. Transcription d'une interview de Theodore Conrad, 4 avril 1956, Avery Architectural and Fine Arts Library, Archives Theodore Conrad. 


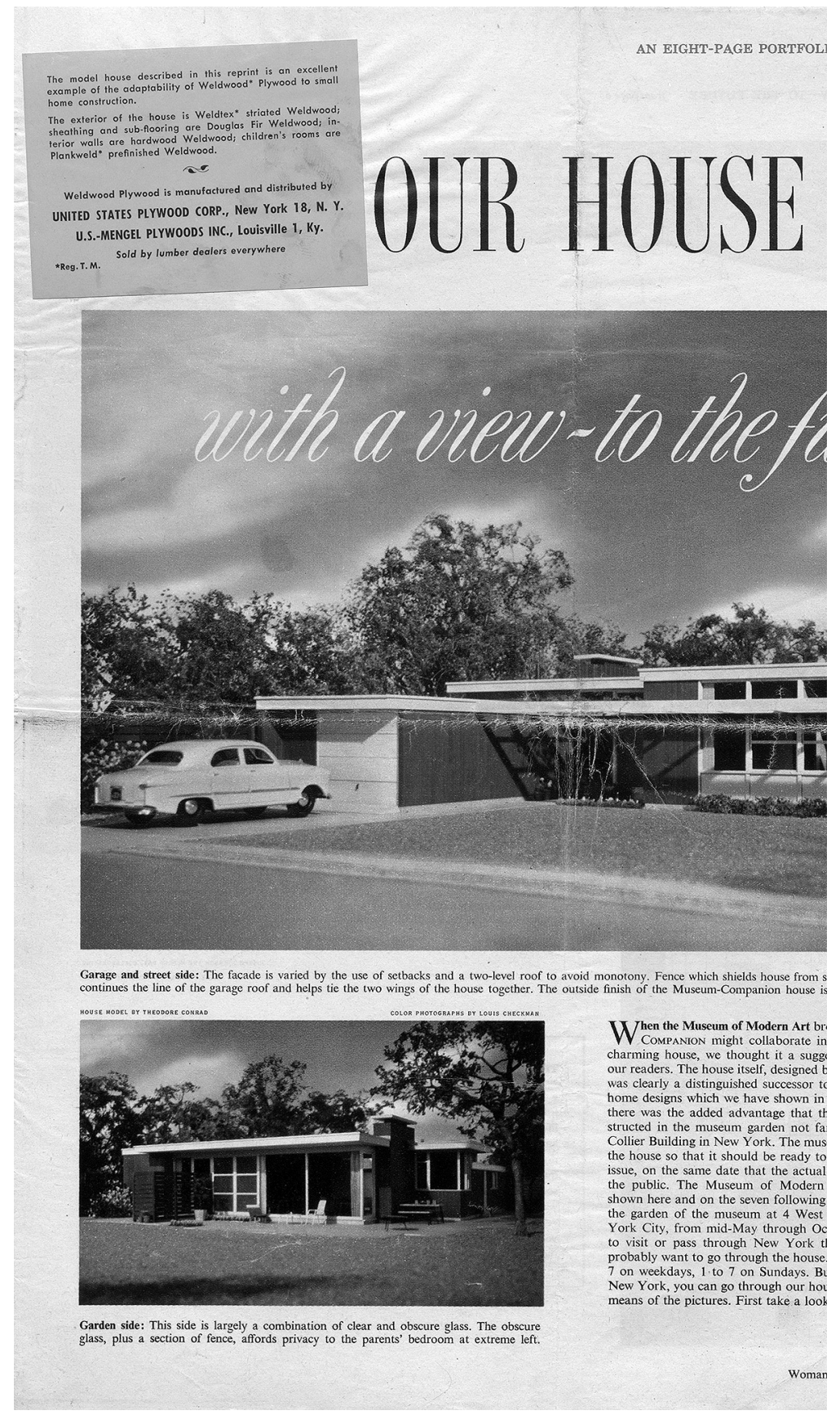

Ill. 4 : Maison pour le jardin du Museum of Modern Art de New York par Gregory Ain, publiée dans Woman's Home Companion en juin 1950, p. 65. (C) Woman's Home Companion. 
développements d'après-guerre, tandis que la presse architecturale était considérablement plus conservatrice dans son choix de dispositifs médiatiques. Pour les maquettistes tels que Conrad, cette ère des magazines fut un âge d'or ${ }^{14}$.

La forte augmentation de l'utilisation des photographies de maquettes était en partie due au besoin désespéré d'illustrations demandées par de nombreux magazines en temps de crise. Pendant la dépression et la guerre, alors que les constructions réelles étaient rares, les magazines tentaient de remplir leurs pages en organisant des concours ou en chargeant directement des architectes de présenter des idées de maisons unifamiliales. Ce furent ces publications qui s'approprièrent alors la photographie de maquette et poussèrent vers un réalisme plus développé afin de séduire leurs lecteurs. Un grand nombre de projets modernistes pour des « maisons du futur" non réalisées présentaient des innovations telles que des plans libres, de grandes fenêtres ouvrant sur des jardins et des équipements tels que des garages intégrés, la climatisation et le chauffage central. Presque toujours conçus sans site précis, les magazines incluaient des plans qui pouvaient être achetés pour un prix compris entre 1 et 3 dollars. Illustrant souvent des fictions architecturales, les photographies produisaient une simulation idyllique de maisons habitées qui se prétendaient "aussi réelles et belles que dans la vraie vie» ("Real as Life and Just as Beautiful»), pas très différente de ce que Collier's avait promis pour sa maison de verre en 1939. Désormais, des magazines tels que les périodiques dédiés à la vie domestique Ladies' Home Journal, McCall's, Woman's Home Companion, Good Housekeeping et House \& Garden ainsi que des publications plus largement diffusées telles que Collier's, Life et Fortune adoptèrent les photographies de maquettes. Au rang des nouveautés, la couleur apparaît dans la photographie de maquette en raison de la demande des magazines pour des pages en couleur; elle est rendue possible grâce à l'introduction de pellicules couleur améliorées, New Agfacolor et Kodachrome, introduites respectivement en 1935 et $1936^{15}$.

Pendant la guerre, Collier's avait interrompu la publication de sa série de maquettes de maisons, pour ne jamais la relancer. Mais la crise du logement était grave dans l'immédiat après-guerre. Désormais, ceux qui continuaient de publier des maquettes de maisons étaient principalement les magazines féminins, ciblant le public qui était censé être le plus touché par la pénurie de logements : les femmes. Woman's Home Companion, publication sœur de Collier's et magazine rencontrant le plus d'écho dans le public féminin, publia chaque mois entre 1947 et 1950 une série de maisons abordables. Sous la tutelle de son rédacteur chargé de la décoration intérieure Harriet Burket et de l'architecte Wallace Heath, le magazine a publié des projets d'aménagement par un certain nombre d'éminents architectes modernes. Les maquettes étaient conçues suivant le précédent de la maison de verre, mais étaient construites à une échelle encore plus grande de $1 / 2$ pouce pour un pied ${ }^{16}$ (environ 1,27 sur $32 \mathrm{~cm}$ ). L'intention était toujours le plus grand réalisme grâce à des points

14. Jane Jacobs, "The Miniature Boom. A Growing Demand for Precision Models Has Propelled the Architectural Modelmaker into the Machine Age ", The Architectural Forum, mai 1958, p. 109.

15. Ernst Born, Geschichte des Bilderdrucks, Basel, ambripress, 2006, p. 82.

16. Conversation avec Ruth Conrad, 7 juin 2013. 


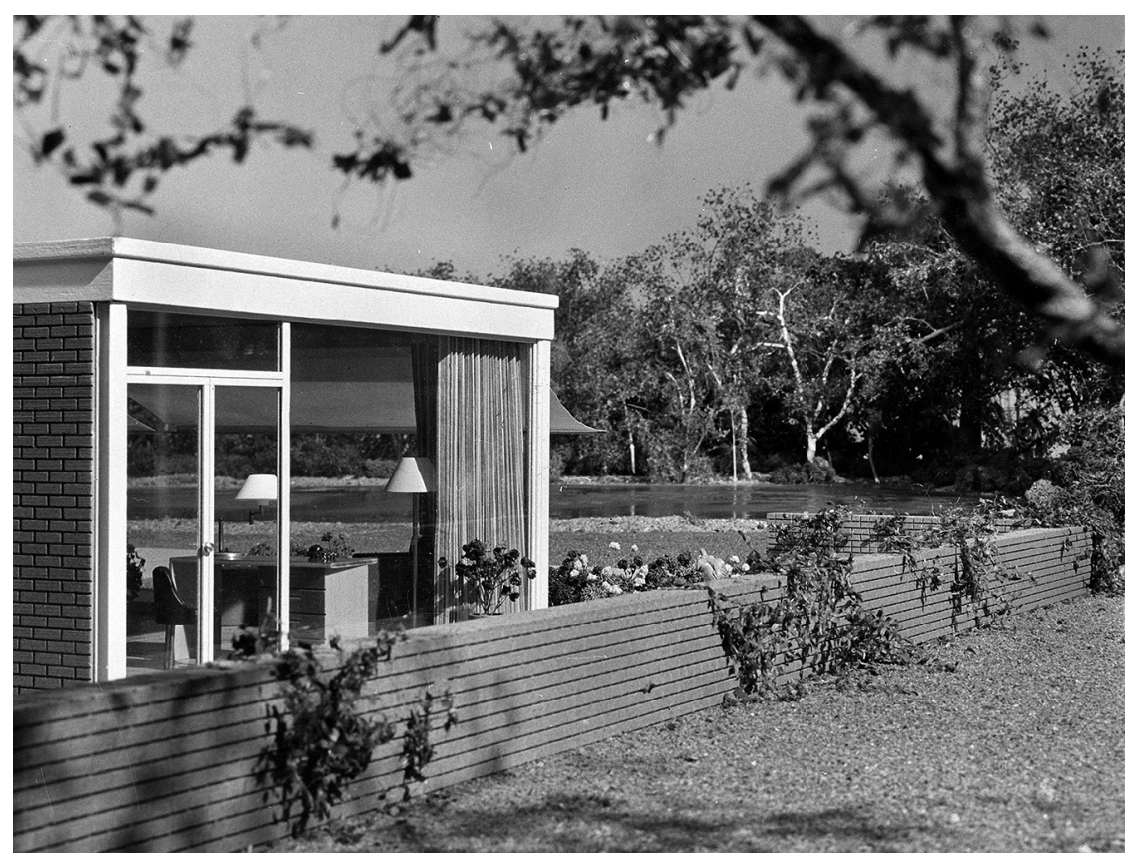

Ill. 5 : Vue rapprochée de la maquette de la maison Speyer. (C) Avery Architectural and Fine Arts Library, Archives Theodore Conrad.

de vue bas, des accessoires élaborés et la simulation de la lumière du soleil ${ }^{17}$. Beaucoup pouvaient être démontées pour des gros plans intérieurs. La grande échelle était une concession au détail, ainsi qu'aux grands formats des chambres photographiques. Comme dans la photographie d'architecture, la plupart des maquettes mettaient en scène un été (fictif), longtemps après la fin de la construction et alors que la végétation avait poussé ${ }^{18}$. Cela signifiait que l'aménagement paysager avait pris une telle importance qu'il prenait environ la moitié du temps consacré à la fabrication de maquettes ${ }^{19}$. Réalisée par Theodore Conrad et photographiée par Louis Checkman, la maquette de la maison Speyer par A. James Speyer (ill. 5) fut publiée en septembre 1948 dans Woman's Home Companion ${ }^{20}$. Sur ces photographies, toutes les innovations d'avant-guerre étaient réunies de manière spectaculaire. Même si

17. Lettre de recommandation de Theodore Conrad à Louis Checkman, 3 novembre 1943, Avery Architectural and Fine Arts Library, Archives Theodore Conrad.

18. Christopher Tunnard fait figure d'exception alors qu'il demanda à ce que la maquette du projet de jardin de 1947 pour le MoMA figure le début du printemps pour mieux faire ressortir les détails architecturaux. Lettre de Christopher Tunnard à Theodore Conrad, 27 août 1946, Avery Architectural and Fine Arts Library, Archives Theodore Conrad.

19. Lettre de Theodore Conrad à Louis Checkman, 29 avril 1945, Avery Architectural and Fine Arts Library, Archives Theodore Conrad.

20. Wallace Heath, "Big as All Outdoors... ", Woman's Home Companion, septembre 1948, p. 62-65. 
aucun chantier de construction n'était envisagé, la maquette était extrêmement détaillée et entièrement meublée. Les nouveaux matériaux de fabrication des maquettes permettaient de produire une image parfaitement réaliste tant de l'intérieur que de l'extérieur grâce aux grandes baies vitrées et au point de vue bas. À travers de grandes illustrations imprimées en couleur, l'intérieur lumineux attirait le regard dans la maison et était utilisé pour des gros plans. Sur les photographies, l'arrière-plan ne laissait aucun espace, cachant le rebord du socle de la maquette et les projecteurs projetaient des ombres réalistes sur la maquette.

\section{Les photographies de maquettes rendues à la conception architecturale}

Au moment de la reprise de la construction après la Seconde Guerre mondiale, les photographies de maquettes réalistes conçues pour donner un aperçu d'un nouveau bâtiment étaient devenues la norme en termes de méthode de publication dans les magazines lifestyle et d'architecture comme dans la presse. L'une des premières photographies de maquettes qui fit la couverture d'une revue d'architecture fut celle prise par Ezra Stoller représentant la maquette réalisée par Theodore Conrad pour les laboratoires de recherche Heinz à Pittsburgh, parue sur la couverture du numéro de mai 1949 d'Architectural Forum (ill. 6). Le Forum avait coopéré avec des magazines lifestyle depuis les années 1930 et, en 1946, était devenu la première publication portant sur l'architecture à éditer des articles illustrés de photographies issues de magazines lifestyle ${ }^{21}$.

La photographie en couleur étant devenue plus importante pour les publications d'intérêt général, les couleurs de la maquette, ainsi que la représentation précise des surfaces, des textures et des matériaux faisaient l'objet d'un examen minutieux au cours du processus de fabrication de la maquette. Cependant, ce furent les publications elles-mêmes qui contribuèrent à développer le réalisme: les flous dans le champ photographique rapproché et l'impression grossièrement tramée des magazines ont aidé à masquer les défauts potentiels qui auraient trahi l'échelle de la maquette. Conscients de cette symbiose parfaite, les rédacteurs en chef ont vu les avantages que présentaient les photographies de maquettes par rapport aux images des bâtiments réels. La possibilité de contrôler les saisons et les conditions météorologiques, ainsi que le recours aux trucs dans la construction de la maquette permettant des vues qui étaient souvent difficiles à obtenir pour les photographies du bâtiment réel, rendaient les photographies de maquettes plus fiables et plus rapides à obtenir. La ressemblance fondamentale entre les photographies de la maquette et celles du bâtiment fini, la « jalousie de la maquette » pour reprendre les termes de

21. John Normile, "Home is Where You Hang Your Mortgage ", The Architectural Forum, avril 1945, p. 91-100 ; Mary Davies Gillies, "Mr. and Mrs. McCall Know What They Want ", The Architectural Forum, avril 1945, p. 101-108. 


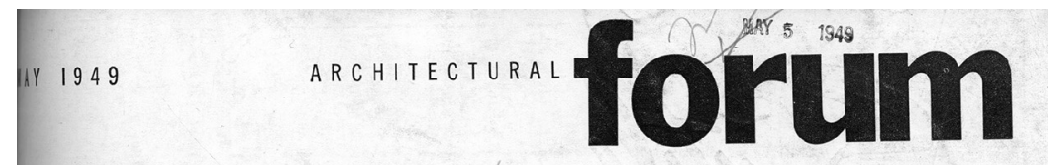

MAGAZINE OF BUILDING

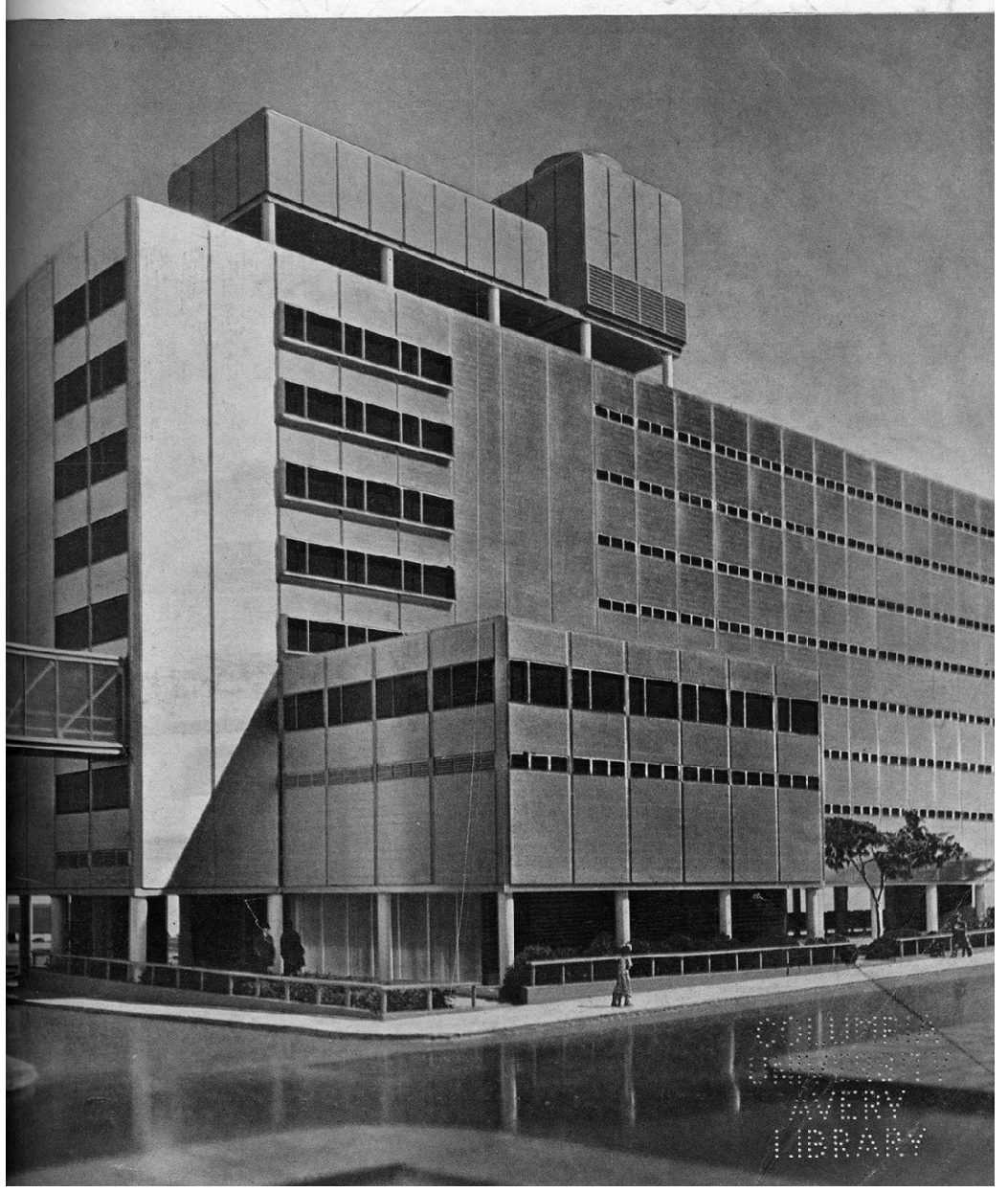

Ill. 6: Photographie de la maquette des laboratoires de recherche Heinz à Pittsburgh par SOM sur la couverture du numéro de mai 1949 d'Architectural Forum. (C) Architectural Forum.

Christian Hubert, avait cependant ses limites ${ }^{22}$. Les figures humaines, qui indiquaient l'échelle, étaient généralement omises car elles auraient détruit l’illusion.

22. Christian Hubert dans Idea as Model, cite par: Morris, "Worlds Collide : Reality to Model to Reality ", Andrew Higgott et Timothy Wray (dir.), Camera Constructs : Architecture and the Modern City, Farnham, Ashgate, 2012, p. 179. 


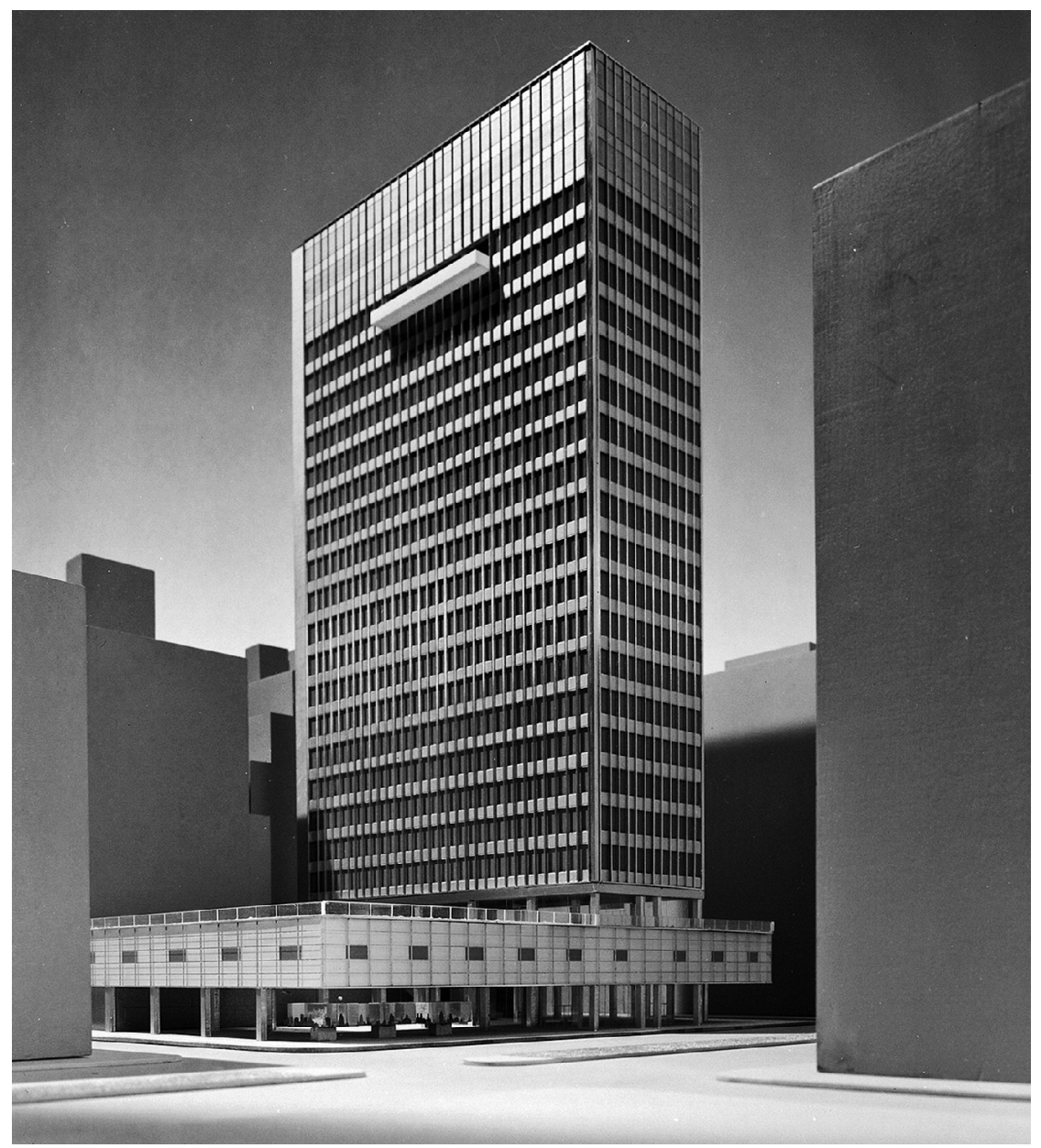

Les architectes devinrent de plus en plus conscients de la nécessité de réaliser des prises de vue de mises en scène réalistes lorsqu'ils commencèrent à utiliser des photographies de maquettes non seulement pour des présentations aux clients, mais aussi dans le cadre du processus de conception. Adoptant des techniques de photographie réaliste dès les premières étapes, un nombre croissant de maquettes furent photographiées comme des bâtiments, depuis des points de vue bas, situés dans un espace urbain identifiable. Même si, comme l'a fait remarquer Daniel Shuster, une maquette vaut idéalement " une centaine d'images ", beaucoup d'entre elles ont été photographiées depuis quelques points de vue définis qui, souvent, anticipaient les vues les plus importantes du bâtiment ${ }^{23}$. Parmi les plus connues, les photographies

23. Lettre de Daniel Shuster à Theodore Conrad, 24 juin 1954, Avery Architectural and Fine Arts Library, Archives Theodore Conrad. 


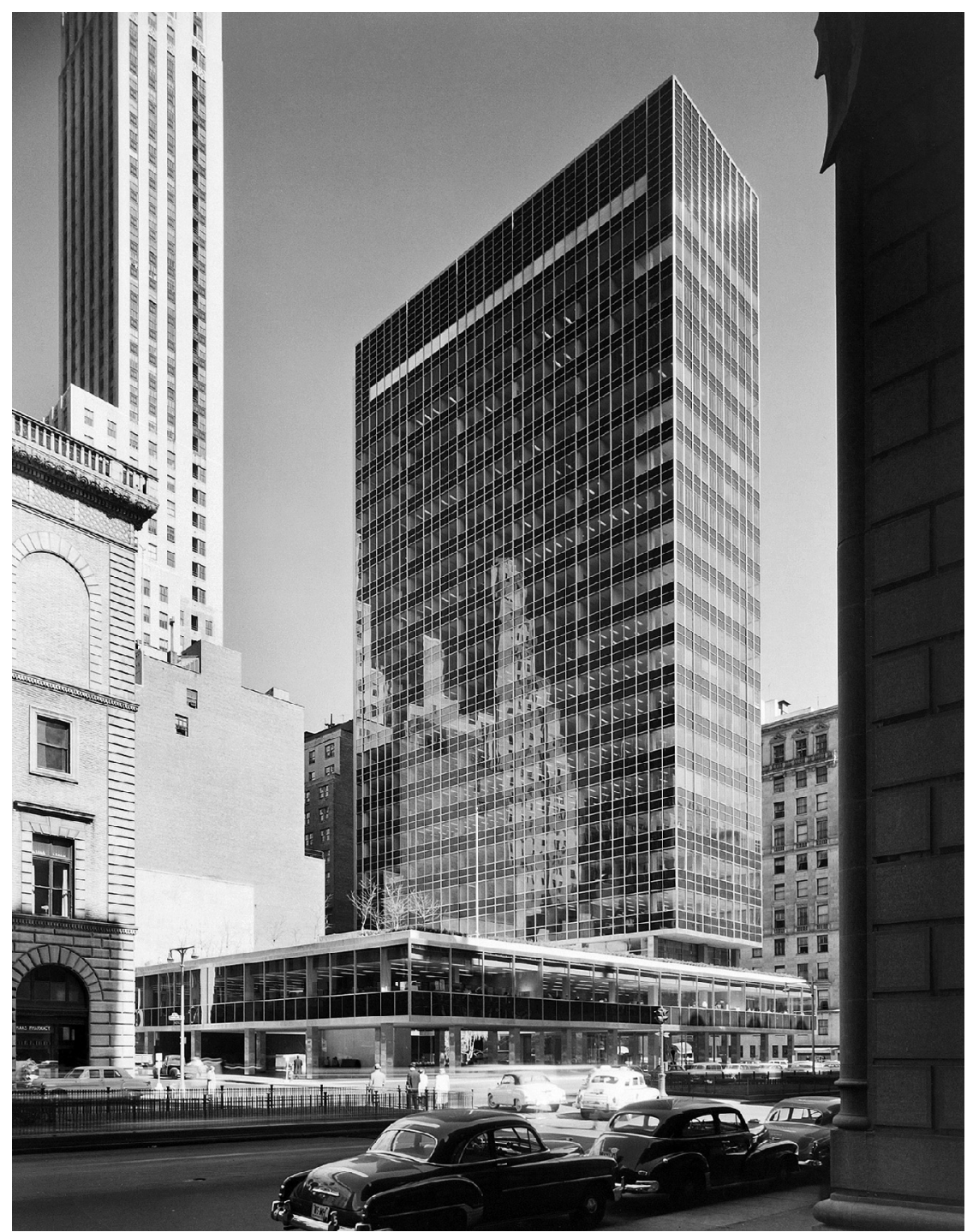

Ill. 7 et 8 : Maquette et bâtiment achevé de la Lever House vus selon un angle similaire. (C) Ezra Stoller/Esto.

d'Ezra Stoller des maquettes de la Lever House de SOM reproduisaient une vue particulière de la structure montrant la proéminence de la tour depuis l'autre côté de Park Avenue - le même point de vue d'où Stoller photographia plus tard le bâtiment fini (ill. 7 et 8). Anticiper les vues d'un bâtiment dans le tissu urbain grâce aux photographies de maquettes permettait de donner au public un aperçu réaliste et autorisait les architectes d'ajuster et d'examiner leurs projets en conséquence. La 
perspective normale de ces photographies est devenue un aspect important de la conception et de la présentation. Plutôt que de créer un environnement fictif pour mettre en valeur un bâtiment imaginé, les maquettes et les photographies devaient désormais mettre à l'essai la manière dont un bâtiment s'intégrait dans un contexte existant. Pour les photographies liées à la conception, l'abondance souvent inutile d'accessoires qui avait caractérisé les photographies de magazine fut abandonnée. Les figures humaines, auparavant laissées de côté, furent introduites pour donner l'échelle du bâtiment. Le virage vers une diffusion plus large des photographies de maquettes durant le boom des miniatures après la guerre fut également visible sur la scène de l'édition architecturale. L'ancien magazine Pencil Points, qui était exclusivement consacré aux rendus, devint Progressive Architecture en 1945, afin de manifester l'abandon du dessin au profit de la photographie d'architecture et de maquette ${ }^{24}$. À ce moment-là, les photographies de maquettes réalistes avaient réussi à convaincre le public de statuer en leur faveur et étaient devenues un support accepté à la fois pour la conception architecturale et pour les présentations publiques. Leur beauté était incontestée.

Teresa FANKHÄNEL Conservatrice à l'Architekturmuseum de la TUM Maîtresse de conférences au département d'histoire de l'architecture et des pratiques curatoriales à l'école d'architecture de la Technische Universität de Munich

24. John Morris Dixon, "Introduction ", George Hartman et Jan Cigliano (dir.), Pencil Points Reader. A Journal for the Drafting Room 1920-1943, New York, Princeton Architectural Press, 2004, XIII. 